\title{
Congenital disorders of glycosylation - an umbrella term for rapidly expanding group of rare genetic metabolic disorders - importance of physical investigation
}

\author{
Lekka DE ${ }^{1,7}$, Brucknerova $\mathrm{J}^{1,7}$, Salingova $\mathrm{A}^{2}$, Sebova $\mathrm{C}^{2}$, Ostrozlikova $\mathrm{M}^{2}$, Ziburova $\mathrm{J}^{3,6}$, \\ Nemcovic $\mathrm{M}^{3}$, Sestak $\mathrm{S}^{3}$, Bellova $\mathrm{J}^{3}$, Pakanova $\mathrm{Z}^{3}$, Sivakova $\mathrm{B}^{3}$, Skoknova $\mathrm{M}^{4}$, Bzduch $\mathrm{V}^{5}$, \\ Mucha $\mathrm{J}^{3}$, Barath $\mathrm{P}^{3}$, Brucknerova $\mathrm{I}^{7}$
}

Faculty of Medicine, Comenius University in Bratislava, Slovakia. ingrid.brucknerova@fmed.uniba.sk

\begin{abstract}
AIM: Congenital disorders of glycosylation (CDG) belong to an expanding group of rare genetic metabolic disorders caused by defects in the complex chemical enzymatic process of glycosylation. The study is aimed at presenting a case report of a premature dysmorphic newborn, clinical presentation of the condition, the way it was diagnosed and treated, as well as its comparison with the known cases.

RESULTS: The result of glycan analysis supports the assumption of a supposed glycosylation disorder and also specifies a specific subtype: CDG-1, subtype ALG12-CDG (Ig).

CONCLUSION: CDG have an extremely wide clinical spectrum and should be considered in any child with unexplained developmental delay, failure to thrive, seizures, and abnormalities in liver enzymes, coagulation and immunologic factors. The treatment of most forms of CDG depends upon numerous factors such as specific symptoms present, severity of the disorder, age and overall health of the patients and tolerance to certain medications or procedures. For these reasons, the treatment is specific for every individual. It is based on the symptoms and requires a coordination of efforts of a team of specialists (Tab. 4, Fig. 3, Ref. 19). Text in PDF www.elis.sk

KEY WORDS: newborn, congenital disorders of glycosylation, antithrombin III.
\end{abstract}

\begin{abstract}
Abbreviations: $\mathrm{CDG}$ - congenital disorders of glycosylation, GPIglycophosphatidylinositol, FCMD - Fukuyama congenital muscular dystrophy, MEB - muscle-eye-brain, IEF - isoelectric focusing
\end{abstract}

\section{Introduction}

Congenital disorders are conditions of prenatal origin that are present at birth and can be structural or functional, occurring in isolation as a single defect or as a group of defects. Approximately $50 \%$ of all congenital disorders do not have a known specific cause and many of them remain of undetermined aetiology. They occur often due to errors in embryogenesis at critical periods of foetal

${ }^{1}$ Faculty of Medicine, Comenius University in Bratislava, Slovakia, ${ }^{2}$ Department of Laboratory Medicine, National Institute of Children's Disease, Bratislava, Slovakia, ${ }^{3}$ Slovak Academy of Sciences, Institute of Chemistry, Department of Glycobiology, Bratislava, Slovakia, ${ }^{4}$ Neonatal Department of Intensive Medicine National Institute of Children's Diseases, Bratislava, Slovakia, ${ }^{5}$ Department of Paediatrics, National Institute of Children's Diseases, Bratislava, Slovakia, ${ }^{6}$ St. Elizabeth Cancer Institute, Department of Clinical Genetics, Bratislava, Slovakia, and ${ }^{7}$ Neonatal Department of Intensive Medicine, Faculty of Medicine, Comenius University in Bratislava, Slovakia

Address for correspondence: I. Brucknerova, MD, PhD, Prof, Faculty of Medicine, Comenius University in Bratislava, NUDCH, Limbova 1, SK-833 41 Bratislava, Slovakia. development or they are attributed to underlying genetic defects or chromosomal abnormalities $(1,2,3,4)$.

Congenital disorders of glycosylation (CDG) belong amongst rare congenital metabolic disorders, with only approximately 1,200 cases diagnosed worldwide. There are many undiagnosed and misdiagnosed CDG patients, making it difficult to determine the true frequency and the real number of cases remains unknown. The disorders affect males and females in equal numbers and in most cases become apparent in infancy. They are caused by defects in the complex enzymatic process of glycosylation which is critical for the development and maturation of the body organs and especially for the nervous system. Glycosylation is the process by which complex oligosaccharides, named glycans, are created, altered and chemically attached either to proteins to make glycoproteins, or to lipids to make glycolipids, with both of them having numerous important functions in all tissues and organs. The process of glycosylation involves many different genes that encode many different proteins such as enzymes. Defects have been found in the activation, presentation, and transport of sugar precursors, in glycosidases and glycosyltransferases involved in glycan synthesis and processing, and in proteins that control the traffic of components of the glycosylation machinery within the cell. A deficiency or lack of one of these enzymes can lead not only to a variety of symptoms that can affect multiple organ sys- 
Tab. 1. Representative biochemical and molecular defects, chromosomal location, number of patients, and year identified (1, 7).

\begin{tabular}{|c|c|c|c|c|c|}
\hline CDG type & Enzymatic or protein defect & Gene & Location & Patients & Year \\
\hline \multicolumn{6}{|c|}{ Group I: Defects in the assembly and transfer of lipid-linked oligosaccharide to proteins in the endoplasmic reticulum } \\
\hline PMM2-CDG (Ia) & Phosphomannomutase 2 & PMM2 & $16 \mathrm{p} 13$ & 300 & 1995 \\
\hline MPI-CDG (Ib) & Phosphomannose isomerase & MPI & $15 \mathrm{q} 22$-qter & 20 & 1998 \\
\hline ALG6-CDG (Ic) & $\begin{array}{l}\text { Dolichyl-P-Glc:Man9GlcNAc2-PP- doli- } \\
\text { chyl } \alpha \text {-1,3-glucosyltransferase }\end{array}$ & ALG6 & $1 \mathrm{p} 22.3$ & 30 & 1999 \\
\hline ALG3-CDG (Id) & $\begin{array}{l}\text { Dolichyl-P-Man:Man5GlcNAc2-PP- doli- } \\
\text { chyl } \alpha-1,3 \text { mannosyltransferase }\end{array}$ & NOT56L & 3 & 2 & 1999 \\
\hline DPM1-CDG (Ie) & Dolichol-P-Man synthase 1 & DPMI & $20 \mathrm{q} 13$ & 4 & 2000 \\
\hline MPDU1-CDG (If) & $\begin{array}{l}\text { Dolichol-P-Man utilization defect } 1 \\
\text { (suppressor of Lec35) }\end{array}$ & MPDUI & $17 \mathrm{p} 12-13$ & 4 & 2001 \\
\hline$\underline{\mathrm{Ix}}$ & Multiple Defects: causes unknown & -------- & & $?$ & \\
\hline \multicolumn{6}{|c|}{ Group II: Defects in the processing of N-glycans } \\
\hline MGAT2-CDG (IIa) & 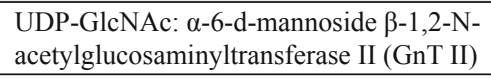 & MGAT2 & $14 \mathrm{q} 21$ & 4 & 1994 \\
\hline GCS1-CDG (IIb) & $\alpha$-1,2-glucosidase I & GCSI & $2 \mathrm{p} 12-13$ & 1 & 2000 \\
\hline SLC35C1-CDG, LAD-II (IIc) & GDP-fucose transporter (cytosol $\rightarrow$ Golgi) & ----------- & None & 4 & 2001 \\
\hline
\end{tabular}

CDG - congenital disorders of glycosylation

Tab. 2. Representative genetic defects in the $\mathrm{O}$-glycosylation pathway related to congenital disorders of glycosylation (12).

\begin{tabular}{lll}
\hline CDG Type & Gene symbol & Protein name \\
\hline EXT1/EXT2-CDG & EXT1/EXT2 & Glucuronyltransferase/N-acetylglucosaminyltransferase \\
\hline B4GALT7-CDG & B4GALT7 & $\beta$-1,4-galactosyltransferase I $(\beta$ 4GalT7) \\
\hline GALNT3-CDG & GALNT3 & N-acetylgalactosaminyltransferase \\
\hline SLC35D1-CDG & SLC35D1 & UDP-GlcA/UDP-GalNAc Golgi dual transporter \\
\hline POMT1/POMT2-CDG & POMT1/ POMT2 & O-mannosyltransferase 1/O-mannosyltransferase 2 \\
\hline POMGNT1-CDG & POMGNT1 & O-mannosyl- $\beta$-1,2-N-acetylglucosaminyltransferase \\
\hline SCDO3-CDG & LFNG & O-fucose-specific $\beta$-1,3-N-acetylglucosaminyltransferase \\
\hline B3GALTL-CDG & B3GALTL & $\beta$-1,3-glucosyltransferase specific for O-fucose-linked glycans on thrombospondin type 1 repeats \\
\hline
\end{tabular}

CDG - congenital disorders of glycosylation

tems and almost always include an important neurological component, but also to a variety of severities from mild cases to severe and disabling or life-threatening cases. Most often children will display abnormalities in liver enzymes, digestion and absorption, glandular function, blood clotting, and immunologic function and should be considered in any child with unexplained developmental delay, failure to thrive, seizures, abnormalities in liver enzymes, coagulation and immunologic factors $(5,6,7)$. The diagnosis in most cases can be done by a simple blood test, analysing the glycosylation status of transferrin. The treatment of these disorders depends on the particular variation of clinical presentation and symptomatology of each patient $(1,8,9,10,11)$.

The classification and pathophysiology of congenital disorders of glycosylation can be seen in Tables 1 and 2 .

An incorrect synthesis of oligosaccharides in both glycoproteins and glycolipids results in broad multisystem clinical manifestations, as they have very important biologic functions. Almost all types of congenital disorders of glycosylation present in infancy. The clinical presentation of a patient may or may not include one or more of the following: failure to thrive, developmental delay, hepatopathy, hypotonia, neurologic abnormalities, hypoglycaemia, protein-losing enteropathy, eye abnormalities, immunologic findings, skin abnormalities, and skeletal findings. It is becoming clear that the clinical spectrum can involve individual or multiple organ systems and may or may not affect neurodevelopment. For many types of CDG, the phenotype is not completely known because only a few affected individuals have been reported $(1,4,5,7,10,11,12,13)$.

The most frequent type of CDG is CDG-Ia or PMM2 deficiency, accounting for $70 \%$ of CDG cases, with the prevalence of $1: 20,000$ and estimated number of more than 700 patients known worldwide $(3,4,9,14)$. It affects many systems of the body and mainly the nervous system. Often, patients can be diagnosed in the neonatal or early infantile period based on typical clinical features: inverted nipples, hypotonia, almond-shaped eyes with strabismus, large forehead, unusual distribution of body fat and body pads, abnormal genitalia, failure to thrive, elevated transaminases, osteopenia and cerebellar hypoplasia. There is a substantial childhood mortality of approximately $25 \%$, due to severe infections or organ failure. At a later age, the impairment of the neurologic system becomes more evident with a variable degree of mental retardation, cerebellar dysfunction, and retinitis pigmentosa. Some children experience seizures or exhibit stroke-like episodes while neuro imaging may demonstrate an enlarged cisterna magna and superior cerebellar cistern, which can correlate with functional and cognitive assessments, myelination that varies from normal to insufficient, or delayed maturation and areas of ischaemia or oedema followed by 


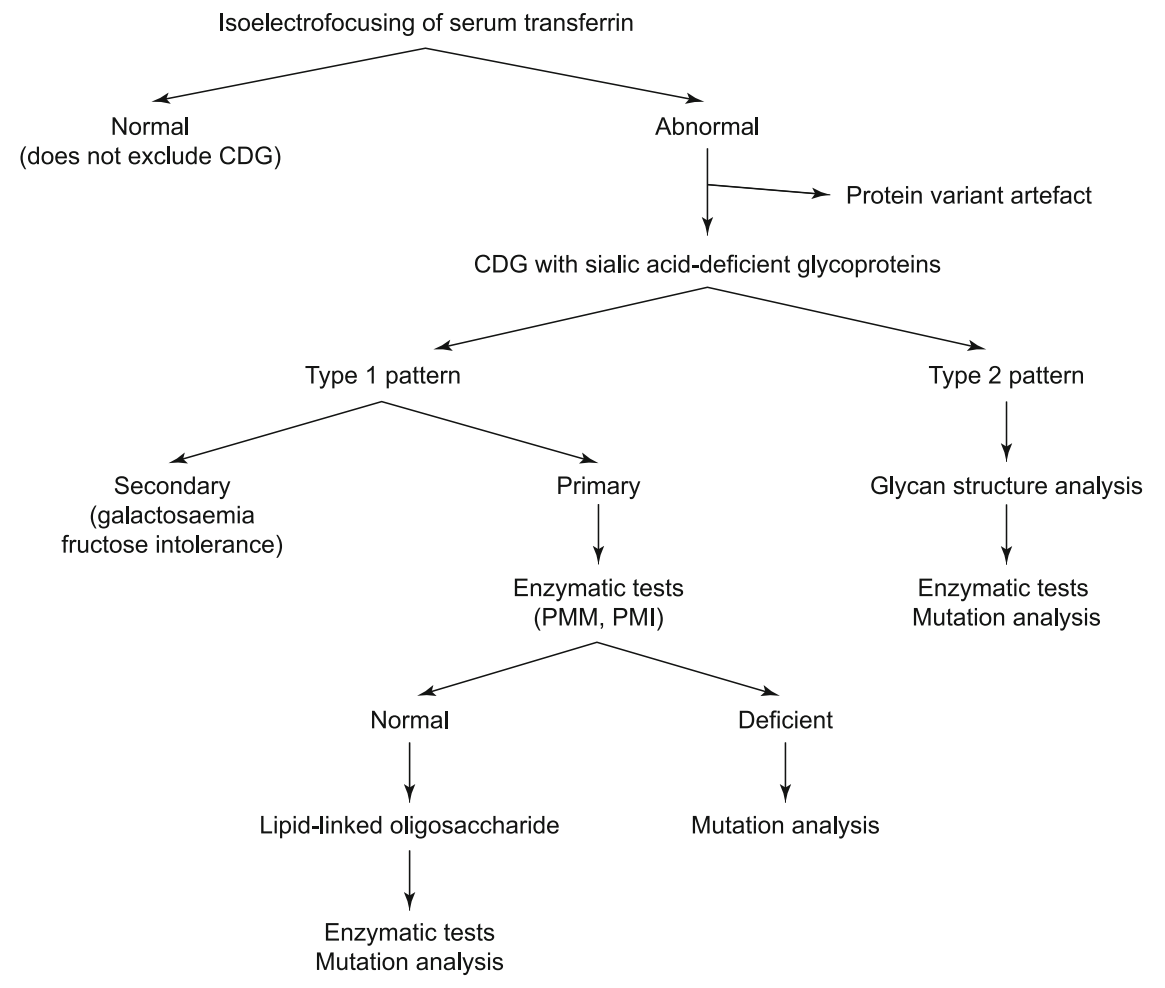

Fig. 1. Diagnostic flowchart for congenital disorders of N-linked glycosylation (13). CDG - congenital disorders of glycosylation, PMM phosphomannomutase, PMI - phosphomannose isomerase.

focal necrosis. In adults, nonprogressive ataxia, stable mental retardation, and peripheral neuropathy mainly characterize the disease. Adult female patients present usually with hypergonadotropic hypogonadism and do not reach sexual development. The disorder impairs both the ability to move physically and coordinate that movement. For this reason, most of the patients are wheelchair-bound throughout their life and are unable to live independently $(1,3,4$, $5,6,9,10,13)$.

Many different metabolic disorders appear with similar signs and symptoms as CDG. These disorders include mitochondrial disorders, myopathies, urea cycle disorders, inborn errors of bile metabolism, fatty acid oxidation disorders, organic acidurias, peroxisome biogenesis disorders, and sphingolipidoses (Tab. 3) (1, 15).

As demonstrated in Figure 1, the first diagnostic step of all $\mathrm{N}$-linked types of CDG is the analysis of serum transferrin glycoforms, also called "transferrin isoforms analysis" or "carbohydrate-deficient transferrin analysis." This diagnostic test is performed by isoelectric focusing (IEF) or capillary electrophoresis, CE-ESI-MS or MALDI-MS, and it can determine the presence and number of incomplete sialylated N-linked oligosaccharide residues linked to serum transferrin. IEF allows the separation of molecules such as proteins or enzymes based upon their electrical charge, thus allowing to detect abnormal serum transferring. Even though negative results cannot exclude CDG, abnormal results can help us decide how to proceed to further investigational tests in order to determine the exact type of CDG and gene that is involved (Tab. 4) $(1,13,4,6)$.
The treatment of most forms of CDG depends upon numerous factors such as the specific symptoms present, severity of the disorder, age and overall health of the patients, as well as tolerance to certain medications or procedures. For these reasons, the treatment of every individual is specific. It is based on the symptoms and requires a coordination of efforts of a team of specialists $(1,4,5,10,11,13)$.

Although the treatment of most forms of CDG is only symptomatic, there are three disorders that have an existing therapy. MPI-CDG (CDG-Ib), which is characterized by hepatic-intestinal disease, is the most common type of CDG for which therapy exists. Patients with MPI-CDG are treated with oral mannose, 4-6 doses per day $\mathrm{x} 100-150 \mathrm{mg} / \mathrm{kg}$ per day. This treatment has been shown to achieve significant improvement while bypassing the underlying genetic defect in glycosylation that causes the disorder $(1,4$, $5,11,13)$.

Some individuals with SLC35C1-CDG (CDG-IIc) have been treated with oral fucose supplementation, $25 \mathrm{mg} / \mathrm{kg}$ per day in three doses. This therapy depends upon the nature of the underlying mutation of the SLC34C1 gene. It improves the fucosylation of glycoproteins and can be beneficial in treating recurrent infections associated with this form of CDG and achieving health improvement. However, fucose therapy does not help with other symptoms of this disorder $(1,4,5,11,13)$. Lastly, butyrate treatment in individuals with PIGM-CDG helps in managing the seizures associated with this form of CDG, namely by increasing the transcription of PIGM. 
A possible therapy for CDG is being studied based on replacing the missing enzyme in cases with deficiency or lack of a particular enzyme. For that reason, synthetic versions of missing enzymes have been developed and used in the treatment of individuals with a certain form of a related group of disorders known as the lysosomal storage diseases (6).

Furthermore, gene therapy, where the defective gene is replaced with a normal gene in order to produce the active enzyme, is also being studied and theoretically is most likely to lead to a cure. However, at this point in time, there are still many technical difficulties to be resolved before the gene therapy can succeed (6).

On the other hand, for individuals who have residual enzyme activity, and thus a milder disease expression, methods to boost or improve any residual enzyme activity are examined in order to further decrease the symptom severity and progression (6).

It is also being studied whether simple pharmacological agents can be developed that would bypass the underlying genetic defect in CDG, while allowing proper glycosylation to occur. Such agents may increase the synthesis or activity of alternative enzymes that could carry out the functions that are normally performed by the deficient enzymes (6).

The premature dysmorphic newborn (wide root of the nose, long philtrum, lower hair boarder, lower positioned ears, smaller neurocranium, flat occipital bone, atypical finger crossing (Figs 2 and 3), pectus excavatus, squared butt, hypogonadism, undescended testicles) was a second child from biamniales bichoriales twins (Caesarean section at the 34th week of gestation; birth weight 2,100 grams; birth length $44 \mathrm{~cm}$; head circumference $30 \mathrm{~cm}$; value of Apgar score $6 / 7 / 10$ points). After birth, severe oedema, signs of respiratory insufficiency (mechanical ventilation, administration of surfactant), multiple skin petechiae, thrombocytopaenia, hypocoagulation status (fresh frozen plasma, antithrombin III), hypoglycaemia, conjugated hyperbilirubinaemia and pedes equinovari were present.

The patient was admitted to our department at the age of 9 days and had a 19-day ventilator support. He developed an adnate infection presented as bronchopneumonia and was treated with antibiotics. The newborn had problems tolerating enteral feeding, he did not gain weight. Mild signs of necrotizing enterocolitis were confirmed. Echocardiography confirmed presence of persistent opened ductus arteriosus and dilatated aortic root. Computed
Tab. 3. Differential diagnosis of congenital disorders of glycosylation (12).

\begin{tabular}{|c|c|c|}
\hline $\begin{array}{l}\text { Types of Congenital } \\
\text { disorders of glycosylation }\end{array}$ & Disorders & Specific examples \\
\hline \multirow{8}{*}{ Most common } & Myopathies & \\
\hline & Disorders of metabolism & \\
\hline & Urea cycle disorders & \\
\hline & Inborn errors of bile & \\
\hline & Fatty acid oxidation disorders & \\
\hline & Organic acidurias & \\
\hline & Peroxisome biogenesis disorders & \\
\hline & Sphingolipidoses & \\
\hline \multirow{9}{*}{$\begin{array}{l}\text { Phosphomanno-mutase } 2 \\
\text { (PMM2-CDG or } \\
\text { CDG- Ia) }\end{array}$} & Cerebral palsy & \\
\hline & Prader-Willi syndrome & \\
\hline & Congenital coagulation disorders & \\
\hline & \multirow{2}{*}{ Congenital myopathies } & X-linked myotubular myopathy \\
\hline & & Multiminicore disease \\
\hline & \multirow{3}{*}{ Congenital muscular dystrophies } & $\begin{array}{l}\text { Fukuyama congenital muscular } \\
\text { dystrophy }\end{array}$ \\
\hline & & Muscle-eye-brain \\
\hline & & Walker-Warburg syndrome \\
\hline & $\begin{array}{l}\text { Ataxia-telangiectasia and other } \\
\text { hereditary ataxias }\end{array}$ & \\
\hline \multirow{5}{*}{$\begin{array}{l}\beta-1,4 \text {-galactosyl- } \\
\text { transferase I } \\
(\text { B4GALT7-CDG) }\end{array}$} & Hypermobility Spectrum disorders & \\
\hline & Occipital horn syndrome & \\
\hline & Loeys-Dietz syndrome & \\
\hline & $\begin{array}{l}\text { Familial hypermobility } \\
\text { syndrome }\end{array}$ & \\
\hline & Marfan syndrome & \\
\hline \multirow{3}{*}{ EXT1/EXT2 } & Hereditary multiple exostoses & \\
\hline & Metachondromatosis & \\
\hline & $\begin{array}{l}\text { Langer-Giedion/trichorhinophalan- } \\
\text { geal syndrome type II }\end{array}$ & \\
\hline
\end{tabular}

Tab. 4. Diagnosis of congenital disorders of glycosylation (12).

\begin{tabular}{ll}
\hline TEST & REASON \\
\hline Isoelectric focusing of serum transferring & $\begin{array}{l}\text { Determine the presence and number of incomplete si- } \\
\text { alylated N-linked oligosaccharide residues linked to } \\
\text { serum transferring }\end{array}$ \\
\hline Blood groups & Identify the Bombay blood group for fucose defects \\
\hline Enzyme assay & Measure the activity of a specific type of enzyme \\
\hline PMM and MPI activity measurement & Identify enzyme function \\
\hline Molecular genetic testing & Identify gene mutations \\
\hline Glycan structure analysis & Identify the responsible enzymes and genes \\
\hline
\end{tabular}

PMM - phosphomannomutase, MPI - phosphomannose isomerase

aortography was negative. Anaemia was twice corrected by transfusion of erythrocytes. Our patient has prolonged deficiency of antithrombin III, factor IX and XI without clinical signs of haemorrhage. Haemophilia was not confirmed. In differential diagnosis of conjugated hyperbilirubinaemia together with elevated hepatic parameters and hypocholic stool, we excluded the bile plug and congenital abnormality of the bile system. The patient had negative serology (cytomegalovirus, varicella, Ebstein-Barr virus, herpes virus, hepatitis B, hepatitis A). From skeletal abnormalities we confirmed the presence of right-sided pes equinovarus and left metatarsus adductus. Metabolic investigation did not confirm galactosaemia, hereditary intolerance of fructose and cystic fibrosis. 


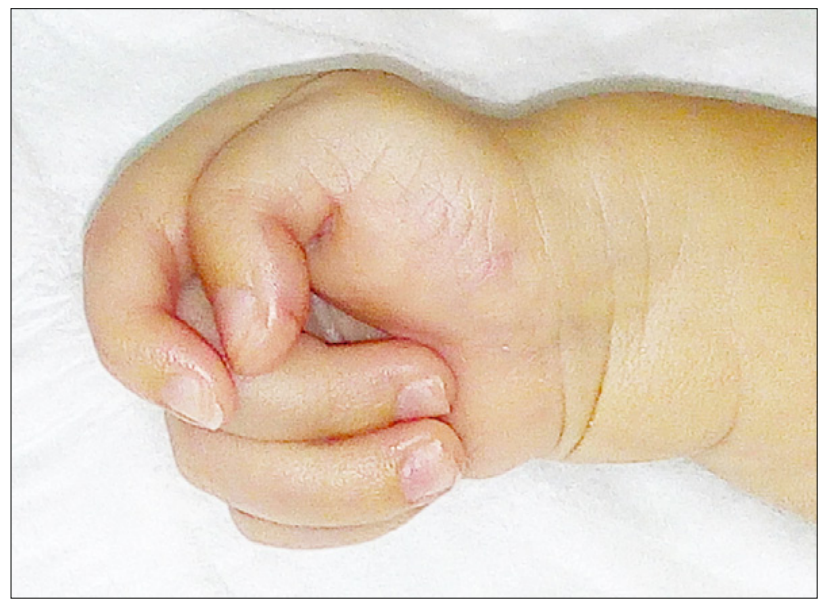

Fig. 2. Atypical finger crossing of the left hand.

Based on many dysmorphic features, nephropathy (dilatation of grade I on the right kidney and of grade II on the left kidney), hepatopathy, hypopotassaemia, hyponatraemia, repeated infections, and skeletal abnormalities, the investigation of a congenital disorder of glycosylation was indicated. Type I N-glycosylation was suspected due to the repeated positive result of the CDG screening. The TLC detection of mono- and disaccharides revealed a distinctive spot of glucose and galactose in accordance with the quantitative determination of both analytes. A slightly increased excretion of galactitol in the urine was captured (embryotoxon was negative). The result of glycan analysis supports the assumption of a supposed glycosylation disorder and also specifies a specific subtype, namely CDG-1, subtype ALG12-CDG (Ig).

Neurological investigation did not confirm abnormalities of the brain. He was not able to swallow and had axial hypotonia and hypertonia on extremities at 7 weeks of age (12).

\section{Discussion}

The wide variety of signs and symptoms in ALG12-CDG are likely due to impaired glycosylation of proteins and lipids that are needed for normal function of many organs and tissues, including the brain $(16,17,18)$. The ALG12 defect is characterized by an accumulation of LLO DolPP-GlcNAc $\mathrm{Man}_{7}$ oligosaccharide in the endoplasmic reticulum. The gene mutations lead to the production of an abnormal mannosyltransferase VIII enzyme with reduced activity, resulting in incomplete oligosaccharides, as mannose cannot be added to the chain efficiently $(16,17,18)$.

The congenital disorder of glycosylation, type Ig or ALG12$\mathrm{CDG}$, is an inherited disorder that can affect several body systems and present with a different clinical picture in each patient. Up to this date, only 9 ALG12-CDG patients were reported worldwide. The first signs and symptoms of the condition usually appear during infancy and can present as feeding problems, failure to thrive, delayed development, intellectual disability and hypotonia. Furthermore, patients can have dysmorphic facial features such as epicanthic folds, prominent nasal bridge and abnormally shaped ears,

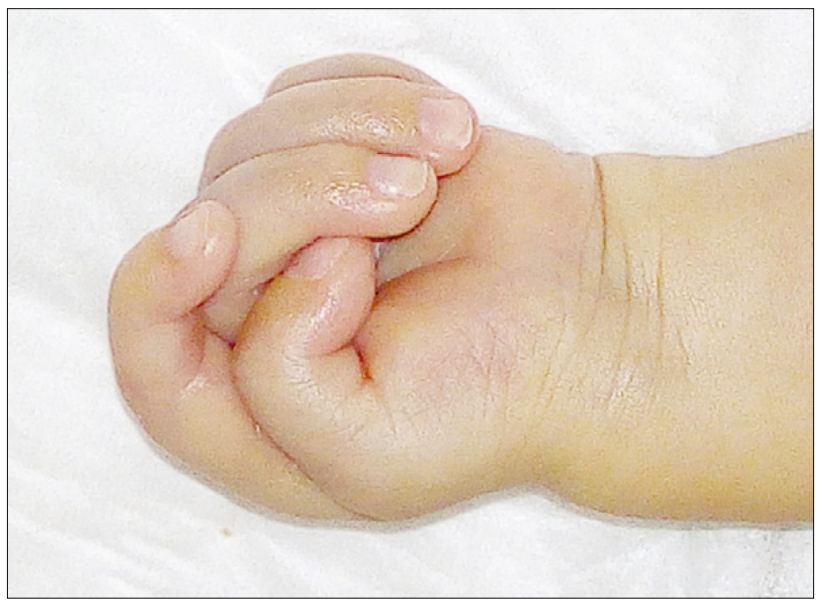

Fig. 3. Atypical finger crossing of the right hand.

but also hypogonadism or hypospadias and undescended testicles. Also, it is common to suffer from frequent upper respiratory tract infections, impaired immunity with decreased immunoglobulin levels and decreased coagulation factors. Skeletal abnormalities and cardiomyopathy features are less common $(16,17,18)$.

The clinical characteristic of our patient shares many similarities with the common clinical presentation of patients with ALG12CDG. Our patient had ventilator support after delivery and administration of surfactant was necessary. Because of the worsening of breathing and persistent retention of $\mathrm{pCO}_{2}$, the newborn was intubated under artificial ventilation regime to SIMV. Frequent upper respiratory tract infections are a common feature in patients with CDG-Ig, and our patient, after chest X-rays showing dystelectasis at the left cardiac side and positive finding of haemolytic strain of E. coli in cultures, was also diagnosed with bronchopneumonia and treated with antibiotics, particularly with cefotaxime.

Feeding difficulties, which belong to the most typical signs of CDG-Ig, were identified also in our patient during infancy. The patient had diet intolerance. Mother's milk was therefore replaced with an artificial complete hydrolysed milk formula, which he tolerated well. The maintenance of appropriate nutrition and sufficient caloric intake is critical in new-born infants with CDG, and as in our case, its achievement can be a challenging endeavour (1, $3,4,5,6,9,10,13)$.

There were no clinical findings of bleeding manifestations that were expected in that kind of disorder, deficiencies in antithrombin III, factor IX and factor XI were found.

Due to the clinical signs of the patient and findings of dysmorphic features, polystigmatisation, nephropathy, hepatopathy, antithrombin III deficiency, hypoglycaemia, hypopotassaemia and hyponatriemia, frequent upper respiratory infections, impaired immunity and undescended testes, a congenital disorder of glycosylation was suspected, an extensive diagnostic metabolism screening was performed and DNA was isolated. Further, an echocardiographic examination was performed with a negative finding, except for a persistent ductus arteriosus up to $1 \mathrm{~mm}$ with a left-to-right shunt and good chamber function. Cardiomyopa- 
thy can appear in CDG-Ig patients, but is not a common feature $(16,17,18)$.

Skeletal abnormalities are also not common for CDG-Ig. Because of the presence of talipes equinovarus (pes equinovarus) on the lower limbs in our patient, an X-ray of the skeleton was performed. Except for pectus excavatum and a metatarsus adductus I sin., Xrays of the spine and other bones were without structural changes.

The first step in identifying the mutations in patients suspected with CDG is sequence analysis. Biochemically, pathological isoelectric transferrin was detected, which is a result of glycosylation type 1 . The final diagnosis of the patient is a congenital disorder of glycosylation, type Ig, or ALG12- CDG.CDG-Ig.

During the whole stay at the hospital, the patient had treatment according to the current signs and symptoms presented at the time, as there is no specific treatment for CDG-Ig. The treatment requires a multidisciplinary coordination of efforts of specialists. Generally, the specific therapeutic procedures and interventions for individuals with CDG varies and depends on numerous factors including the specific symptoms present, severity of the disorder, individual's age and overall health, and tolerance to certain medications or procedures. The clinical manifestations of various types of CDG often differ. The prognosis cannot be predicted in all patients. It is possible, even for individuals with the same type of CDG, not to have the same clinical symptoms and prognosis (1, $3,4,5,6,9,10,12,13,16)$.

\section{Conclusion}

Congenital disorders of glycosylation is an umbrella term for a rapidly expanding group of rare genetic metabolic disorders due to defects in the chemical process of glycosylation. During recent years, the field of CDG has been rapidly expanding because of the increasing awareness of clinical variability of CDG and use of yeast genetic technology. The extremely wide clinical spectrum of CDG makes a broad screening for these disorders in children as well as in adults mandatory by using serum transferrin IEF. It is quite certain that many new glycosylation disorders are awaiting their identification since it is estimated that more than 500 genes, approximately 0.5 to $1 \%$ of the translated human genome, participate in oligosaccharide synthesis and function. For that reason, defects in glycosylation pathway need to be considered more often as the cause of these disorders and physicians need to be aware of them, know their limitations and appropriate way of management (19). Furthermore, the development of animal models offers the potential to test therapeutic approaches and will be helpful in understanding the pathogenesis of these disorders (6).

\section{References}

1. Freeze HH. Update and perspectives on congenital disorders of glycosylation. Glycobiology 2001; 11 (12): 129R-143R.

2. Freeze HH, Schachter H, Kinoshita T. Genetic Disorders of Glycosylation. $3^{\text {rd }}$ edition. Essentials of Glycobiology. Cold Spring Harbor Laboratory Press; 2015-2017. 2015.
3. Losfeld ME, Ng BG, Kircher M, Buckingham KJ, Turner EH, Eroshkin A, Freeze HH. A new congenital disorder of glycosylation caused by a mutation in SSR4, the signal sequence receptor 4 protein of the TRAP complex. Hum Mol Genet 2014; 23 (6): 1602-1605.

4. What is CDG- CDG SOCIETY. (n.d.).

5. Albahri Z. Congenital Disorders of Glycosylation: A Review. Am J Pediatr 2015; 1 (2): 6-28.

6. Nyhan WM. Multiple Carboxylase Deficiency. (Biotinidase Deficiency). In: NORD Guide to Rare Disorders. Lippincott Williams \& Wilkins. Philadelphia, PA. 482. 2003.

7. Snow TM, Woods CW, Woods AG. Congenital Disorder of Glycosylation: a case report. Adv Neonat Care 2012; 12: 96-100.

8. Cylwik B, Lipartowska K, Chrostek L, Gruszewska E. Congenital disorders of glycosylation. Part II. Defects of protein o-glycosylation. Acta Biochim Pol 2013; 60 (3): 361-368.

9. Jaeken J. Congenital disorders of glycosylation. Handb Clin Neurol 2013; 113: 1737-1743.

10. Sparks SE, Krasnewich DM. Congenital Disorders of N-Linked Glycosylation and Multiple Pathway Overview. GeneReviews( $\left({ }^{\circledR}\right)$. [bookaccession]. 2014.

11. Jaeken J, Matthijs G. Congenital disorders of glycosylation. Annu Rev Genomics Hum Genet 2001; 2: 129-151.

12. Lekka DE. Congenital disorders of glycosylation. Diploma thesis. 2018; 72 .

13. Grünewald S, Matthijs G, Jaeken J. Congenital disorders of glycosylation: A review. Pediatr Res 2002; 52 (5): 618-624.

14. Freeze HH, Chong JX, Bamshad MJ, Ng BG. Solving glycosylation disorders: Fundamental approaches reveal complicated pathways. Am J Hum Genet 2014; 94 (2): 161-175.

15. Adam MP, Ardinger HH, Pagon RA, et al. GeneReviews ${ }^{\circledR}$ [Internet]. Seattle (WA): University of Washington, Seattle; 1993-2018. 2018. ISSN: 2372-0697.

16. Grubenmann CE, Frank CG, Kjaergaard S, Berger EG, Aebi M, Hennet T. ALG12 mannosyltransferase defect in congenital disorder of glycosylation type lg. Hum Mol Genet 2002; 11 (19): 2331-2339.

17. Chantret I, Dupré T, Delenda C, Bucher S, Dancourt J, Barnier A, Charollais A, Heron D, Bader-Meunier B, Danos O, Seta N, Durand G, Oriol R, Codogno P, Moore SE. Congenital disorders of glycosylation type Ig is defined by a deficiency in dolichyl-P-mannose:Man7GlcNAc2PP-dolichyl mannosyltransferase. J Biol Chem 2002; 12 (28): 2581525822. Epub 2002 Apr 30.

18. Thiel C, Schwarz M, Hasilik M, Grieben U, Hanefeld F, Lehle L, von Figura K, Körner C. Deficiency of dolichyl-P-Man:Man7GlcNAc2PP-dolichyl mannosyltransferase causes congenital disorder of glycosylation type Ig. Biochem J 2002; 1 (Pt 1): 195-201.

19. Yerlikaya FH, Can U, Alpaydin MS, Aribas A. The relationship between plasma microRNA and serum trace elements levels in primary hyperlipidemia. Bratisl Med J 2019; 120 (5): 344-348.

Received March 6, 2020. Accepted October 7, 2020. 